\title{
Thyroid-Stimulating Activity and Chorionic Gonadotropin
}

\author{
Bruce C. Nisula and Jean-Marie Ketelslegers \\ From the Reproduction Research Branch, National Institute of Child Health \\ and Human Development, National Institutes of Health, \\ Bethesda, Maryland 20014
}

A BSTRACT The nature of the substance with thyroid-stimulating activity (TSA) present in human chorionic gonadotropin (hCG) prepared from pregnancy urine was investigated. In the mouse thyrotropin bioassay, the characteristic maximum of blood radioactivity obtained with the TSA in hCG preparations occurred after that obtained with pituitary thyrotropin (hTSH) but before that obtained with long-acting thyroid stimulator. Antiserum to the $\alpha$ subunit of hCG produced significant neutralization of the TSA in hCG. Significant antagonism of hTSH biologic activity was achieved with certain doses of hCG, suggesting that the TSA in hCG was a partial agonist of hTSH. This antagonism was neutralized by antiserum to the $\boldsymbol{\beta}$ subunit of hCG. These immunologic results suggest that the substance with TSA in hCG preparations contains antigenic determinants similar to those of both the $\alpha$ and the $\beta$ subunit of hCG. Amounts of highly purified hCG and crude commercial hCG of equal immunologic activity were biologically indistinguishable in the bioassay for TSA. Both hCG immunoreactivity and the TSA in hCG adsorbed to concanavalin $\mathrm{A}$ and eluted with $0.2 \mathrm{M}$ methyl $\alpha$-D-glucopyranoside. These results are consistent with the hypothesis that TSA is an intrinsic property of hCG or of a glycoprotein molecule physicochemically, biologically, and immunologically similar to hCG.

\section{INTRODUCTION}

The available evidence suggests that clinically significant modification of thyroid gland activity in the human can occur in response not only to pituitary thyrotropin $(\mathrm{hTSH})^{1}$ and long-acting thyroid stimulator (LATS)

Received for publication 14 December 1973 and in revised form 31 January 1974.

${ }^{1}$ Abbreviations used in this paper: bTSH, bovine pituitary thyrotropin; hCG, human chorionic gonadotropin; $\mathrm{hCG} \alpha, \alpha$ subunit of chorionic gonadotropin; hCG $\beta, \beta$ subunit of chorionic gonadotropin; hTSH, human pituitary thyrotropin; LATS, long-acting thyroid stimulator; $\mathrm{MeG}$, methyl $\alpha$-D-glucopyranoside; TSA, thyroid-stimulating activity; $\mathrm{TSH}$, thyroid-stimulating hormone. but also to a substance with thyroid-stimulating activity (TSA) secreted by trophoblastic tissues. For instance, increased thyroid function and occasionally overt hyperthyroidism have been observed in women with trophoblastic diseases (1-6) and in men with testicular tumors that secrete chorionic gonadotropin (hCG) $(7,8)$. These clinical observations have stimulated attempts to isolate and characterize the substance with TSA secreted by trophoblastic tissues. In previous studies, TSA has been found in some extracts of placenta (9-14), in mole tissue (15), in pregnancy serum extracted by the method of Bates $(14,16)$, and in hCG preparations from normal pregnancy urine (10). The latter observation in concert with the suggestion that folliclestimulating activity could be an intrinsic property of hCG (17) prompted consideration of the hypothesis that TSA is an intrinsic property of hCG. Thus the current investigation was undertaken to understand the nature of the TSA found in association with hCG and, in particular, to determine if some of the currently available physicochemical and immunological methods can distinguish the hCG from the substance with TSA in $\mathrm{hCG}$ preparations.

\section{METHODS}

A commercially available urinary hCG preparation (Lot No. 372311 of Pregnyl, Organon, Inc., West Orange, N. J.) was used except where indicated. Highly purified hCG (CR117), derived from commercially available urinary hCG, was a gift of Drs. Francis J. Morgan and Robert E. Canfield, College of Physicians and Surgeons, Columbia University, New York (18). During the purification procedure the hCG specific biologic activity increases about fivefold (18). The hTSH preparation (hTSH 68/38) was kindly provided by the Medical Research Council, Mill Hill, London. The serum of a patient with Graves' disease was the source of the LATS. The method used to prepare the mice for bioassay of TSA has been described elsewhere (19). Blood samples $(100 \mu \mathrm{l})$ were obtained immediately before injection of the test material intraperitoneally (initial) and at 2,9 , and $22 \mathrm{~h}$ after injection. The response variable was calculated as the ratio of 2,9 , or $22 \mathrm{~h}$ to initial counts times $100 \%$. Potency estimates were calculated by the method of Brownlee (20), and the $95 \%$ fiducial 


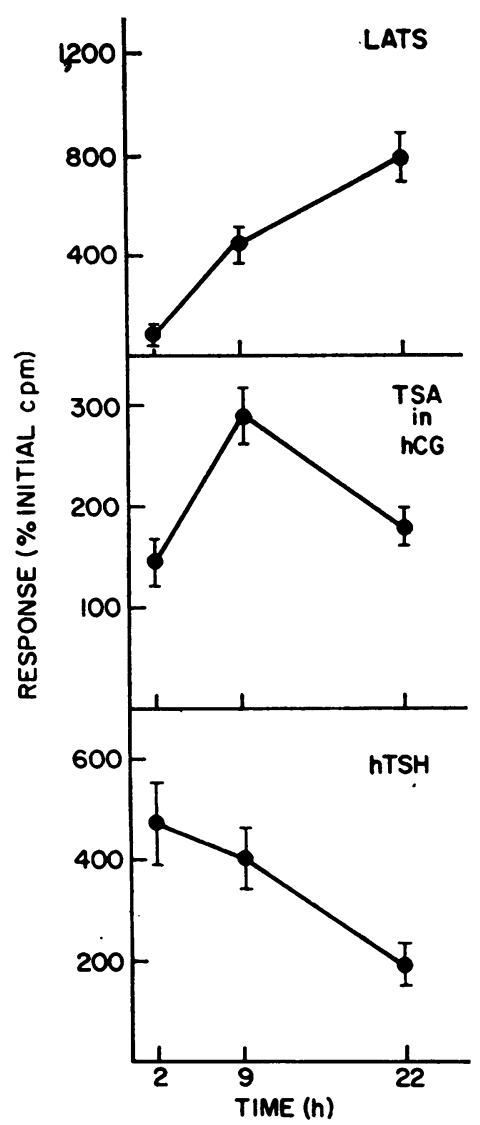

Figure 1 Time course of the response obtained in the mouse thyrotropin bioassay with intraperitoneal injection of $0.5 \mathrm{ml}$ serum containing LATS, the TSA in $500 \mathrm{IU}$ of hCG, and $0.5 \mathrm{mU}$ hTSH. Closed circles and brackets indicate the mean and $\mathrm{SE}$ of the responses of five mice.

limits were analyzed by the method of Finney (21). Criteria for significant neutralization of biologic activity were those described previously (19).

For the biologic comparison of the TSA of highly purified hCG (CR117) and commercial hCG (Pregnyl Lot No. 372311), equivalent amounts of immunoreactive hCG were used to generate the dose-response curves.

Adsorption of TSA in hCG to concanavalin A and elution with methyl $\alpha$-D-glucopyranoside (MeG) (Calbiochem, San Diego, Calif.) were assessed. Approximately 30,000 IU hCG were incubated at room temperature with $30 \mathrm{ml}$ Con-A Sepharose (Pharmacia Fine Chemicals, Uppsala, Sweden $)$ in phosphate buffer $\left(0.1 \mathrm{M} \mathrm{PO}_{4}, 0.15 \mathrm{M}\right.$ $\mathrm{NaCl}, \mathrm{pH}$ 7.8). The mixture was centrifuged, and the sediment was extracted first with $0.2 \mathrm{M} \mathrm{MeG}$ and then 1.0 M MeG. Extracts were dialyzed, lyophilized, reconstituted in phosphate buffer, radioimmunoassayed for hCG, and bioassayed for TSA.

Since the substance with TSA in hCG seemed to be a less potent thyroid stimulator than hTSH, we looked for some antagonism of the biologic activity of hTSH by the substance with TSA in the hCG preparation. To demonstrate this effect, increasing amounts of hCG were admixed with a fixed amount of hTSH $(0.5 \mathrm{mU})$, and the bioassay response was measured $2 \mathrm{~h}$ after intravenous injection of the test solutions.

The anti-hCG $\alpha(\mathrm{hCG} \alpha)$ and anti-hCG $\beta(\mathrm{hCG} \beta)$ sera used in these studies were generated in rabbits. The immunologic properties of these antisera have been described elsewhere $(19,22)$. The hCG binding capacities of the anti-hCG $\alpha$ and anti-hCG $\beta$ sera were about 8,000 and 770 IU hCG/ml, respectively, by Scatchard analysis (23). Initial attempts to produce neutralization of the TSA in hCG were performed by measuring the TSA of a mixture of $\mathrm{hCG}$ and anti-hCG $\alpha$ serum. The resulting mixture was not well tolerated by the mice; although there was substantial reduction in the response, variances were unusually large, and the neutralization was not considered significant. Thus, binding of the substance with TSA in hCG to anti-hCG $\alpha$ serum was assessed by another method. $2 \mathrm{ml}$ of either anti-hCG $\alpha$ serum or nonimmune serum were admixed with $15,000 \mathrm{IU}$ hCG and incubated for $24 \mathrm{~h}$ at $4^{\circ} \mathrm{C}$. Then, sheep anti-rabbit gamma globulin serum was added to precipitate the immune complex. TSA remaining in the supernate was measured in the mouse thyrotropin bioassay.

Because of the scarcity and the relatively low binding capacity of the anti-hCG $\beta$ serum, only indirect assessment of its immunologic cross-reactivity with the substance with TSA in hCG could be performed. Thus, the ability of the anti-hCG $\beta$ serum to neutralize one of the properties of the substance with TSA in hCG (i.e. antagonism of hTSH biologic activity), apparent at relatively low doses of the hCG preparation, was determined by admixing a dose of the hCG preparation antagonistic to hTSH with hTSH and the anti-hCG $\beta$ serum.

\section{RESULTS}

The characteristic bioassay responses obtained with hTSH, the TSA in hCG, and LATS administered intraperitoneally are shown in Fig. 1 . The maximal response to hTSH was observed at $2 \mathrm{~h}$ and to LATS at 22 $h$; whereas the maximal response to the TSA in hCG was observed at $9 \mathrm{~h}$. This type of intermediate response was also obtained with Bates extracts of crude hCG and

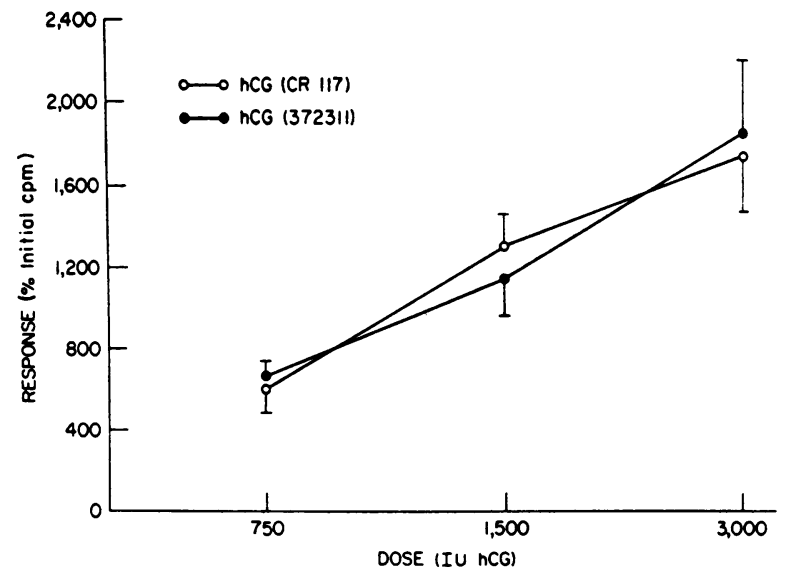

FIGURE 2 Dose-response relationship of crude commercial hCG (Pregnyl Lot No. 372311) and highly purified hCG (CR117) in the mouse thyrotropin bioassay. Symbols and brackets indicate the mean and SE of the 9-h responses of five mice. 


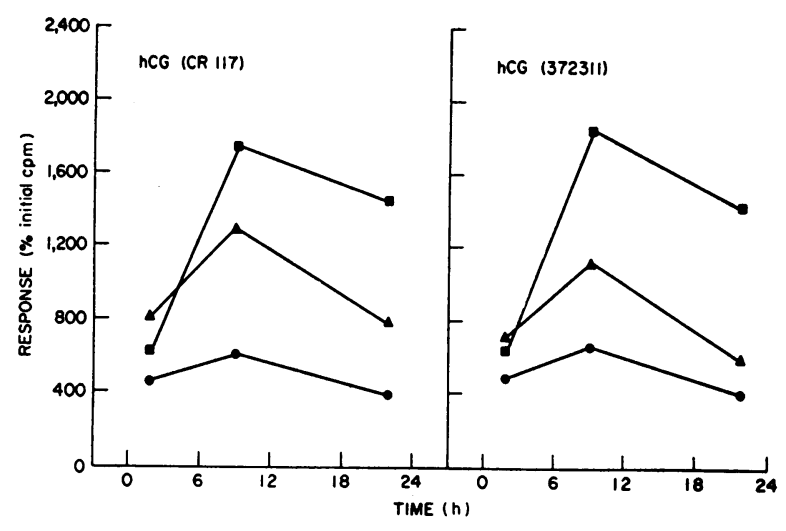

Figure 3 Time course of the response obtained in the mouse thyrotropin bioassay with highly purified hCG (CR117) and crude commercial hCG (Pregnyl Lot No. 372311). Each symbol indicates the mean response of five mice given $750 \mathrm{IU}$ (circles), 1,500 IU (triangles), or 3,000 IU (squares) hCG.

the unextracted serum and kaolin-acetone urine extracts of patients with molar pregnancy and hCG-producing testicular tumor. No response was obtained with a dose of $0.5 \mathrm{ml}$ of a pool of unextracted pregnancy serum (8-12th wk of pregnancy) containing $54 \mathrm{IU}$ hCG $/ \mathrm{ml}$.

Equivalent amounts of immunoreactive, highly purified hCG (CR117) and crude hCG (372311) produced doseresponse curves in the mouse thyrotropin bioassay that were indistinguishable in slope and potency (Fig. 2). The point estimate of the potency ratio was $0.95(95 \%$ fiducial limits $=0.45-2.05$ ). From Fig. 3, the similarity of the time courses of the responses for these two hCG preparations is obvious.

Adsorption of both the hCG and the TSA in the hCG

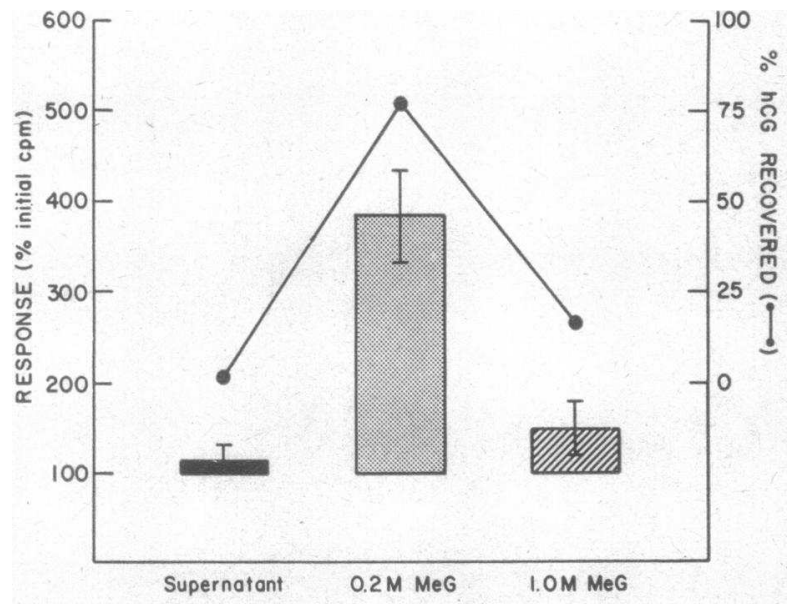

FIGURE 4 TSA and hCG immunologic activity in the supernate and in the $\mathrm{MeG}$ extracts of the hCG preparation adsorbed to concanavalin $\mathrm{A}$. Bars and brackets indicate the mean and $\mathrm{SE}$ of the responses of five mice.

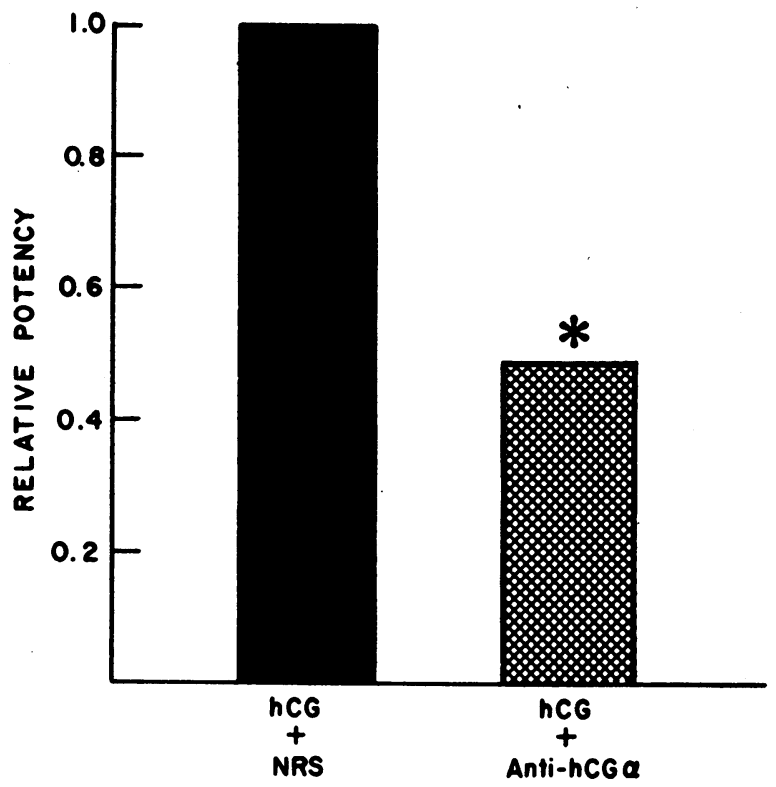

FIGURE 5 Effect of anti-hCG $\alpha$ serum on relative potency of the TSA in hCG in the mouse thyrotropin bioassay. ${ }^{*}$ Indicates significant $(P<0.025)$ reduction in potency. NRS, nonimmune rabbit serum.

preparation to concanavalin A was found (Fig. 4), since essentially no immunologic $\mathrm{hCG}$ or biologic TSA were found in the supernate. Subsequent extraction with 0.2 $\mathrm{M} \mathrm{MeG}$ resulted in recovery of most of the $\mathrm{hCG}$ and TSA in hCG with relatively little further recovered in the $1.0 \mathrm{M} \mathrm{MeG}$ extraction.

As shown in Fig. 5 , the anti-hCG $\alpha$ serum neutralized a significant fraction of the TSA in hCG.

The effect of hCG on the biologic activity of hTSH is shown in Fig. 6. As increasing amounts of hCG were added to a fixed amount of hTSH, some inhibition of the hTSH activity was apparent with 200 IU hCG. This inhibition of the biologic activity of hTSH was further quantified by determining the effect of this same amount of $\mathrm{hCG} / \mathrm{mU} \mathrm{hTSH}$ on the relative potency of the hTSH in the bioassay. As seen in Fig. 7, the relative potency of the hTSH preparation was significantly reduced by mixture with hCG. Also shown in Fig. 7 is the neutralization of hTSH by the anti-hCG $\beta$ serum. However, mixture of the same amounts of hCG and anti-hCG $\beta$ serum together with the hTSH not only failed to produce more inhibition but actually produced less inhibition of hTSH biologic activity (Fig. 7). Thus, the antagonism of hTSH biologic activity by the substance with TSA in the hCG preparation was neutralized by anti-hCG $\beta$ serum. Similar amounts of the $\mathrm{hCG}$ and anti-hCG $\beta$ serum given alone or mixed were not sufficient to evoke responses in control mice. 


\section{DISCUSSION}

The results of the present investigation indicate that the TSA in hCG preparations is either an intrinsic property of the hCG molecule or a property of a molecular entity physicochemically similar to hCG. First and foremost, $\mathrm{hCG}$ retains its TSA during purification. Second, it has been shown that during Sephadex G-100 gel filtration, the TSA in hCG preparations is recovered in the same fraction as the hCG (10), suggesting that the substance with TSA in hCG preparations has a molecular size similar to that of hCG. Third, adsorption to concanavalin $\mathrm{A}$ is a specific characteristic of molecules containing certain sugar linkages (24); and glycoproteins including hCG (25) bind to concanavalin A. In the present study, both hCG and the TSA in hCG were adsorbed to concanavalin A and recovered under the same extraction conditions. This suggests that the substance with TSA in hCG preparations, like hCG, is a glycoprotein.

The results of the immunologic studies of the substance with TSA in hCG preparations, suggest that its antigenic determinants are similar or identical to those of hCG. The anti-hCG $\alpha$ serum that neutralized the TSA in hCG in the present study has previously been shown to neutralize the biologic activity of hCG (26). The observations that this antiserum also neutralizes hTSH, hLH, and hFSH $(19,22)$ but not bTSH (19) suggest that this antiserum contains antibodies dihuman $\alpha$ subunit. Hence, the current results indicate rected toward antigenic determinants specific for the that the substance with TSA in the hCG preparations, like hCG, shares an antigenic structure in common with the $\alpha$ subunits of the human pituitary glycoprotein hormones.

Because of the relatively low binding capacity and the scarcity of the anti-hCG $\beta$ serum, it was not practical to determine neutralization of the substance with TSA in $\mathrm{hCG}$ preparations with admixtures of hCG and anti$\mathrm{hCG} \beta$ serum. Hence, we used a property of the substance with TSA in hCG that was demonstrable at relatively low doses of the hCG preparation to assess the similarity of the antigenic determinants of the hCG $\beta$ subunit to those of the substance with TSA in hCG preparations. The phenomenon of partial agonism has been demonstrated for the polypeptide hormones, oxytocin (27), and adrenocorticotropin (28). Some analogues of these hormones contain relatively weak intrinsic biologic activity and are at certain doses antagonistic to the biologic activity of the parent hormone. The results of the present study suggest that the substance with TSA in hCG preparations was a partial agonist of hTSH in the mouse thyrotropin bioassay. The finding that its antagonism of hTSH biologic activity was neutralized by anti-hCG $\beta$ serum suggests

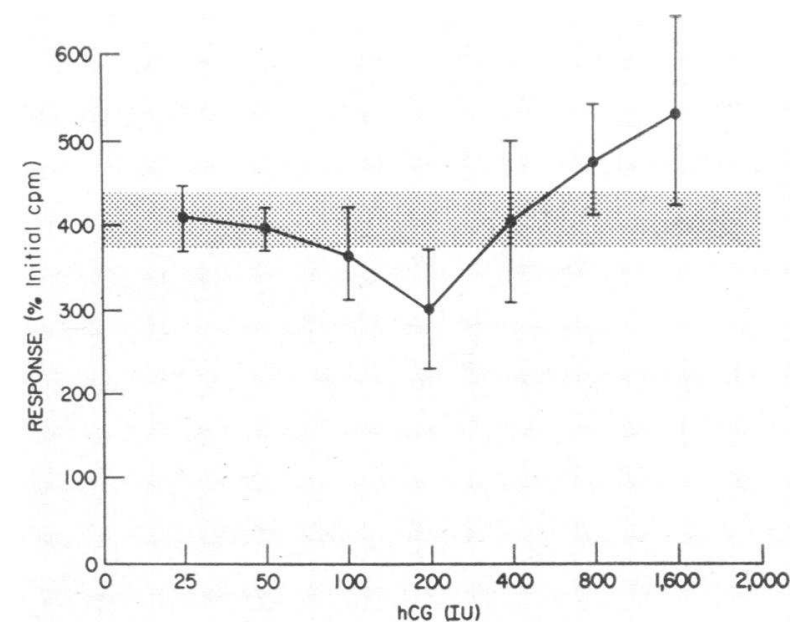

FIGURE 6 Effect of increasing amounts of (hCG) on the bioassay response at $2 \mathrm{~h}$ obtained with a fixed amount of $\mathrm{hTSH}(0.5 \mathrm{mU})$. Shaded area indicates the mean $\pm \mathrm{SE}$ of the 2 -h responses of five mice given $\mathrm{hTSH}$ without hCG.

that the substance with TSA in the hCG preparations has antigenic determinants similar to those of the hCG $\beta$ subunit. That both anti-hCG $\alpha$ and anti-hCG $\beta$ sera crossreact with the substance with TSA in the hCG preparation suggests that it is immunologically similar or identical to hCG. However, it is important to recognize that since highly purified hCG contains TSA, subunits generated from such hCG preparations could be contaminated with a substance or subunits of a substance different from hCG containing this TSA. Antisera generated to subunit preparations could therefore contain antibodies to this hypothetical contaminant. This ex-

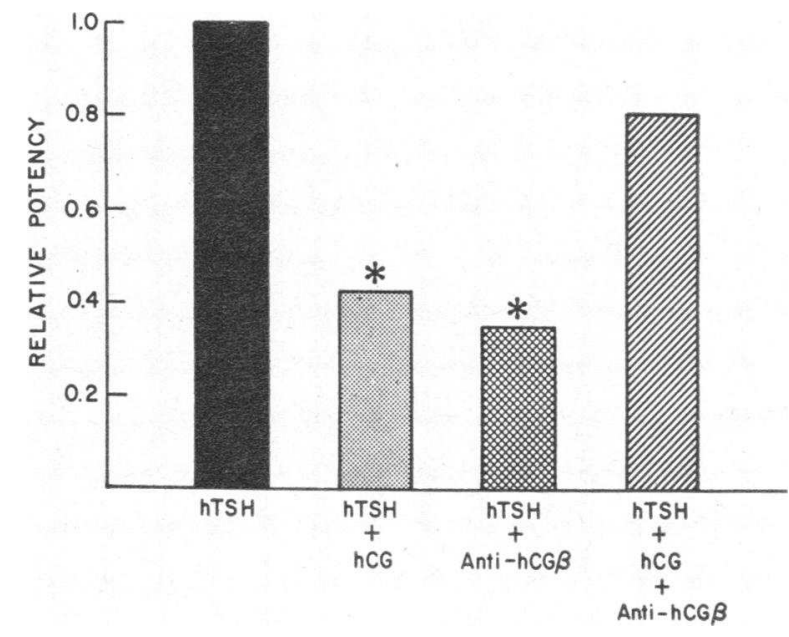

Figcre 7 Effect of hCG, anti-hCG $\beta$ serum, and a mixture of hCG and anti-hCG $\beta$ serum on the relative potency of hTSH in the mouse thyrotropin bioassay. * Indicates significant $(P<0.025)$ reduction in potency. 
planation seems unlikely in light of the purity of the hCG subunits used as immunogen but cannot be excluded.

Since molar tissue contains considerably more hCG than normal placenta $(17,29)$ and $\mathrm{hCG}$ is extracted by the Bates method (17), the observation that Bates extracts of molar tissue (15) but not those of term placenta (10) contain TSA is consistent with the concept that TSA is an intrinsic property of hCG or a closely related entity. Several clinical observations support this concept. First, the levels of serum TSA found during pregnancy parallel the known pattern of serum $\mathrm{hCG}$ levels during pregnancy (14). Second, in a previous study in which 24 -h urine concentrates obtained at various stages of pregnancy were given to hypophysectomized immature female rats, thyrotropic activity paralleled gonadotropic activity (30). Third, all the patients with overt clinical hyperthyroidism presumed due to this stimulator have had hCG levels considerably greater than those usually seen in normal pregnancy (1-8). Fourth, regression of hCG levels is associated with regression of increased thyroid function toward normal (1-7). Although cosecretion of hCG and a similar substance with TSA could be a biologic characteristic of both normal and neoplastic trophoblast, in light of the other evidence it seems more likely that the gonadotropic and thyrotropic activities are characteristics of a single species.

Although our investigation was largely restricted to the study of the TSA in hCG prepared from pregnancy urine, there is substantial evidence indicating that a similar or identical substance with TSA circulates in patients with hCG-secreting tissues (1). In the present study, the intermediate peak response characteristic of the TSA in urinary hCG preparations was also obtained with the unextracted sera of patients with moles and hCG-secreting testicular tumors. Several workers have provided evidence that the substance with TSA found in pregnancy urine circulates in patients bearing hCG-secreting tissues. Bioassay responses more prolonged than that of pituitary thyrotropin were obtained with pregnancy urine extracts (10), extracts of molar tissue (15), and unextracted serum of patients with molar pregnancy (6) and hCG-secreting testicular tumor (8). Further, the substances with TSA in mole serum and mole tissue, pregnancy urine, and serum of a man with an hCG-secreting testicular tumor all eluted during Sephadex G-100 gel filtration well before pituitary TSH and chorionic thyrotropin, the substance with TSA found in Reisfeld, Lewis, Brink, and Steelman (31) extracts of term placenta $(8,15)$. Thus, the substances with TSA found in the serum and urine of patients bearing the various trophoblastic tissues have similar biologic characteristics in the mouse thyrotropin bioassay and are physicochemically different from pituitary TSH as well as from chorionic thyrotropin.

The evidence indicating that there are two substances with TSA associated with trophoblastic tissues has recently been reviewed $(1,32)$. One of these, the chorionic thyrotropin, has been readily detected and characterized only in Reisfeld (31) extracts of placenta (1114). The name "large trophoblastic thyrotropin" was suggested for the other (1) because its apparent molecular size, according to gel filtration studies, was larger than that of chorionic thyrotropin and hTSH (15). The use of this term, large trophoblastic thyrotropin, was suggested for the substance with TSA in hCG preparations from pregnancy urine (1) that appears to be similar or identical to the "molar thyrotropin" found in molar tissue (15) and in the serum of patients with hydatidiform mole (6). It also appears to be similar or identical to the substance with TSA present in the serum of a man with an hCG-secreting testicular tumor (8). Clearly then, our present results suggest that this substance referred to as large trophoblastic thyrotropin is hCG. At the present time, there is not sufficient evidence to resolve whether the chorionic thyrotropin or the TSA in hCG, if either, is responsible for the changes in thyroid function seen in patients with hCG-secreting tissues, although the available evidence, albeit largely circumstantial, appears to implicate the TSA in hCG.

\section{ACKNOWLEDGMENTS}

The authors wish to thank Peter O. Kohler, Judith L. Vaitukaitis, and Griff T. Ross for their encouragement and helpful advice.

\section{REFERENCES}

1. Hershman, J. M. 1972. Hyperthyroidism induced by trophoblastic thyrotropin. Mayo Clin. Proc. 47: 913918.

2. Odell, W. D., R. W. Bates, R. S. Rivlin, M. B. Lipsett, and R. Hertz. 1963. Increased thyroid function without clinical hyperthyroidism in patients with choriocarcinoma. J. Clin. Endocrinol. Metab. 23: 658-664.

3. Dowling, J. T., S. H. Ingbar, and N. Freinkel. 1960. Iodine metabolism in hydatidiform mole and choriocarcinoma. J. Clin. Endocrinol. Metab. 20: 1-12.

4. Cohen, J. D., and R. D. Utiger. 1970. Metastatic choriocarcinoma associated with hyperthyroidism. J. Clin. Endocrinol. Metab. 30: 423-429.

5. Hershman, J. M., and H. P. Higgins. 1971. Hydatidiform mole-a cause of clinical hyperthyroidism. $N$. Engl. J. Med. 284: 573-577.

6. Galton, V. A., S. H. Ingbar, J. Jiminez-Fonseca, and J. M. Hershman. 1971. Alterations in thyroid hormone economy in patients with hydatidiform mole. J. Clin. Invest. 50: 1345-1354.

7. Steigbegel, N. H., J. J. Oppenheim, L. M. Fishman, and P. P. Carbone. 1964. Metastatic embryonal carcinoma of the testis associated with elevated plasma TSH-like activity and hyperthyroidism. N. Engl. J. Med. 271: 345-349. 
8. Karp, P. J., J. M. Hershman, S. Richmond, D. P. Goldstein, and H. A. Selenkow. 1973. Thyrotoxicosis from molar thyrotropin. Arch. Intern. Med. 132: 432436.

9. Akasu, F., S. Kawahara, H. Ohki, M. Harano, and Y. Tejima. 1955. Thyroid-stimulating hormone extracted from human placenta. Endocrinol. Jap. 2: 297-302.

10. Burger, A. 1967. Studies on a thyroid-stimulating factor in urinary chorionic gonadotropin preparations. Acta Endocrinol. 55 : 587-599.

11. Hennen, G. 1965. Detection and study of a humanchorioic-thyroid-stimulating factor. Arch. Int. Physiol. Biochim. 73: 689-695.

12. Hershman, J. M., and W. R. Starnes. 1969. Extraction and characterization of a thyrotropic material from the human placenta. J. Clin. Invest. 48: 923-929.

13. Hershman, J. M., and W. R. Starnes. 1971. Placental content and characterization of human chorionic thyrotropin. J. Clin. Endocrinol. Metab. 32: 52-58.

14. Hennen, G., J. G. Pierce, and P. Freychet. 1969. Human chorionic thyrotropin: further characterization and study of its secretion during pregnancy. J. Clin. Endocrinol. Metab. 29: 581-595.

15. Hershman, J. M., H. P. Higgins, and W. R. Starnes. 1970. Differences between thyroid stimulator in hydatidiform mole and human chorionic thyrotropin. Metab. (Clin. Exp.). 19 : 735-744.

16. Bates, R. W. 1963. The chick method of thyrotropin assay. In Thyrotropin. S. C. Werner, editor. Charles C. Thomas, Publisher, Springfield, Ill. 290-296.

17. Ashitaka, Y., M. Mochizuki, and S. Tojo. 1972. Purification and properties of chorionic gonadotropin from the trophoblastic tissue of hydatidiform mole. Endocrinology. 90: 609-617.

18. Canfield, R. E., F. J. Morgan, S. Kammerman, J. T. Bell, and G. M. Agosto. 1971. Studies of chorionic gonadotropin. Recent Prog. Horm. Res. 27: 121-164.

19. Nisula, B. C., P. O. Kohler, J. L. Vaitukaitis, J. M. Hershman, and G. T. Ross. 1973: Neutralization of human thyrotropin by antisera to subunits of glycoprotein hormones. J. Clin. Endocrinol. Metab. 37: 664-669.
20. Brownlee, K. A. 1960. Statistical Theory and Methodology in Science and Engineering. John Wiley \& Sons, Inc., New York. 291-298.

21. Finney, D. J. 1964. Statistical Methods in Biological Assay. Charles Griffen \& Co. Ltd., London. 2nd edition. 370.

22. Vaitukaitis, J. L., and G. T. Ross. 1973. Recent advances in evaluation of gonadotropic hormones. Annu. Rev. Med. 24: 295-302.

23. Scatchard, G. 1949. The attraction of proteins for small molecules and ions. Ann. N. Y. Acad. Sci. 51: 660-672.

24. Aspberg, K., and J. Porath. 1970. Group-specific adsorption of glycoproteins. Acta Chem. Scand. 24: 18391841.

25. Dufau, M. L., T. Tsuruhara, and K. J. Catt. 1972. Interaction of glycoprotein hormones with agaroseconcanavalin-A. Biochim. Biophys. Acta. 278: 281-292.

26. Rayford, P. L., J. L. Vaitukaitis, G. T. Ross, F. J. Morgan, and R. E. Canfield. 1972. Use of specific antisera to characterize biologic activity of $\mathrm{hCG} \beta$ subunit preparations. Endocrinology. 91: 144-146.

27. Rudinger, J., and I. Krejčí. 1962. Dose-response relations for some synthetic analogues of oxytocin, and the mode of action of oxytocin on the isolated uterus. Experientia (Basel). 18: 585-588.

28. Seelig, S., S. Kumar, and G. Sayers. 1972. Isolated adrenal cells. Proc. Soc. Exp. Biol. Med. 139: 12171219.

29. Diczfalusy, E. 1954. Chorionic gonadotropin and estrogens in the human placenta. Acta Endocrinol. Suppl. 12: 29 .

30. Lyon, R. A., M. E. Simpson, and H. M. Evans. 1953. Qualitative changes in urinary gonadotrophins in human pregnancy during the period of rapid increase in hormone titer. Endocrinology. 53: 674-686.

31. Reisfeld, R. A., U. J. Lewis, N. G. Brink, and S. L. Steelman. 1962. Human growth hormone: preparation from acetone powder. Endocrinology. 71: 559-563.

32. Hershman, J. M., W. R. Starnes, J. G. Kenimer, and R. A. Patillo. 1973. Human chorionic thyrotropin. Int. Congr. Ser. 273: 682-687. 